\title{
Algorithms of the Manipulator Drive Control System
}

\author{
${ }^{1}$ Bulat F. Bairamov, ${ }^{2}$ Artur A. Fardeev, ${ }^{3}$ Albert R. Fardeev \\ Kazan Federal University \\ Email: o.a.fardeeva@mail.ru
}

Received: 20 ${ }^{\text {th }}$ August 2019, Accepted: $30^{\text {th }}$ September 2019, Published: $31^{\text {st }}$ October 2019

\begin{abstract}
The article presents a developed algorithm for the manipulator drive control system using a processor. This algorithm consists of three parts:

- the functioning algorithm of the manipulator drive control system, in order to ensure the movement of the workpiece along a given path;

- the functioning algorithm of the manipulator drive control system, with an aim of eliminating the deviation of the true path of the manipulated object from the set;

- the functioning algorithm of the manipulator drive control system, ensuring the capture of the manipulated object.

When creating the first algorithm, a speed vector control method is used, which consists in reporting specific speeds and accelerations for each of three degrees of mobility, depending on the projections of the trajectory on the coordinate axis and the time required for the manipulation object to pass from the beginning of the path to its end.

When creating the second algorithm, the method of sequential adjustments is used.

The third algorithm is based on a change in the position of the gripper when the generalized coordinates of the workpiece (axial coordinates and rotation angles) go beyond the allowable area.

These algorithms are necessary when creating programs for controlling the manipulator drives, which is used to automate the movement of workpieces in the forging or hot stamping processes.
\end{abstract}

\section{Keywords}

Algorithm, Control System, Manipulator, Predetermined Path, Processor.

\section{Introduction}

When developing the functioning algorithm of the manipulator drive control system, it is necessary to use functional models that provide control for: the movement of the manipulated object along a given path; elimination of the deviation of the true trajectory from the set; bypassing obstacles and capturing the workpiece. These functional models have been previously developed [1].

Based on these models, it is necessary to create algorithms that ensure the operation of manipulator.

\section{Methods}

To compile the manipulator drive control program, ensuring the movement of the workpiece along a given trajectory, based on the functional model developed previously [1], we will draw up an algorithm for the system functioning in fulfilling this goal.

We will use the speed vector control method [2]. The resulting algorithm has the following form:

1) input: $a_{\text {nom }}, v_{\text {nom }}, l_{1}, l_{2}, l_{3}$

2) assignment: $t_{0}:=t ; t_{\mathrm{p}}:=\frac{v_{\text {nom }}}{a_{\text {nom }}} ; t_{\mathrm{T}}:=t_{\mathrm{p}} ; l:=\max \left(l_{1}, l_{2}, l_{3}\right) ; T:=\frac{l}{v_{\text {nom }}}+\frac{1}{2} t_{\mathrm{p}}+\frac{1}{2} t_{\mathrm{T}}$;

$$
k_{\mathrm{p}}:=\frac{t_{\mathrm{p}}}{t} ; k_{\mathrm{T}}:=\frac{t_{\mathrm{T}}}{t}
$$

3) verification of condition fulfillment: $t-t_{0} \leq t_{\mathrm{p}}$;

if the condition is met, go to step 4,5 , then to 3 ;

if the condition is not met, then go to step 6;

4) assignment: $a_{1}:=\frac{l_{1}}{T^{2} k_{\mathrm{p}}\left(1-\frac{1}{2}\left(k_{\mathrm{p}}+k_{\mathrm{T}}\right)\right)} ; a_{2}:=\frac{l_{2}}{T^{2} k_{\mathrm{p}}\left(1-\frac{1}{2}\left(k_{\mathrm{p}}+k_{\mathrm{T}}\right)\right)}$;

$$
a_{3}:=\frac{l_{3}}{T^{2} k_{\mathrm{p}}\left(1-\frac{1}{2}\left(k_{\mathrm{p}}+k_{\mathrm{T}}\right)\right)}
$$

5) Report acceleration $a_{1}, a_{2}, a_{3}$ using appropriate controls

6) verification of condition fulfillment: $t-t_{0} \leq T-t_{\mathrm{p}}$

if the condition is met, go to step 7,8 , then to 6 ;

if the condition is not met, then go to step 9;

7) assignment: $a_{1}:=0 ; a_{2}:=0 ; a_{3}:=0 ; v_{1}:=\frac{l_{1}}{T\left(1-\frac{1}{2}\left(k_{\mathrm{p}}+k_{\mathrm{T}}\right)\right)}$;

$$
v_{2}:=\frac{l_{2}}{T\left(1-\frac{1}{2}\left(k_{\mathrm{p}}+k_{\mathrm{T}}\right)\right)} ; v_{3}:=\frac{l_{1}}{T\left(1-\frac{1}{2}\left(k_{\mathrm{p}}+k_{\mathrm{T}}\right)\right)}
$$

8) Report acceleration $a_{1}, a_{2}, a_{3}$ and speed $v_{1}, v_{2}, v_{3}$ using appropriate controls

9) verification of condition fulfillment: $t-t_{0} \leq T$ 
if the condition is met, go to step 10,11 , then to 9 ;

if the condition is not met, then go to step 12;

$$
\text { 10) assignment: } \begin{aligned}
& a_{1}:=-\frac{l_{1}}{T^{2} k_{\mathrm{T}}\left(1-\frac{1}{2}\left(k_{\mathrm{p}}+k_{\mathrm{T}}\right)\right)} ; a_{2}:=-\frac{l_{2}}{T^{2} k_{\mathrm{T}}\left(1-\frac{1}{2}\left(k_{\mathrm{p}}+k_{\mathrm{T}}\right)\right)} \\
& a_{3}:=-\frac{l_{3}}{T^{2} k_{\mathrm{T}}\left(1-\frac{1}{2}\left(k_{\mathrm{p}}+k_{\mathrm{T}}\right)\right)}
\end{aligned}
$$

11) Report acceleration $a_{1}, a_{2}, a_{3}$ using appropriate controls

12) assignment: $a_{1}:=0 ; a_{2}:=0 ; a_{3}:=0 ; v_{1}:=0 ; v_{2}:=0 ; v_{3}:=0$

13) Report acceleration $a_{1}, a_{2}, a_{3}$ and speed $v_{1}, v_{2}, v_{3}$ using appropriate controls

Where $\boldsymbol{a}_{\text {nom }}, \boldsymbol{v}_{\text {nom }}$ - rated acceleration and speed of processor controls (controller); $\boldsymbol{l}_{\mathbf{1}}, \boldsymbol{l}_{\mathbf{2}}, \boldsymbol{l}_{\mathbf{3}}$ - projections of the trajectory onto the corresponding coordinate axes; $t_{0}$ - initial time; $t$ - current time; $t_{\mathrm{p}}-$ acceleration time; $t_{\mathrm{T}}-$ braking time; $T$ - time of the manipulated object movement from the start point to the end; $k_{\mathrm{p}}, k_{\mathrm{T}}-$ acceleration and braking factors; $\boldsymbol{a}_{\mathbf{1}}, \boldsymbol{a}_{\mathbf{2}}, \boldsymbol{a}_{\mathbf{3}}-$ acceleration projections on the corresponding coordinate axes; $\boldsymbol{v}_{\mathbf{1}}, \boldsymbol{v}_{\mathbf{2}}, \boldsymbol{v}_{\mathbf{3}}-$ speed projections on the corresponding coordinate axes.

Formulas for calculating such quantities as $T, k_{\mathrm{p}}, k_{\mathrm{T}}$, as well as projections of speed and acceleration vectors at the stages of acceleration, motion and braking, used in this algorithm, were obtained earlier [3].

This algorithm is designed for a straight path. Straightness is achieved due to the same acceleration, braking and movement time of the manipulated object across all degrees of mobility [4], as well as due to constant speeds across all degrees of mobility in the movement section [5].

If the presence of an obstacle or process conditions do not allow moving along a straight path, then one can use a broken path with its own speeds and accelerations in each straight section [6].

\section{Results and Discussion}

To create the manipulator drive control program that ensures the workpiece returns to a given path when the true path deviates from the given path based on the functional model previously developed [1], we will draw up an algorithm for the system functioning in solving this problem. We will use the method of successive adjustments [2]. The resulting algorithm has the following form:

1) input:

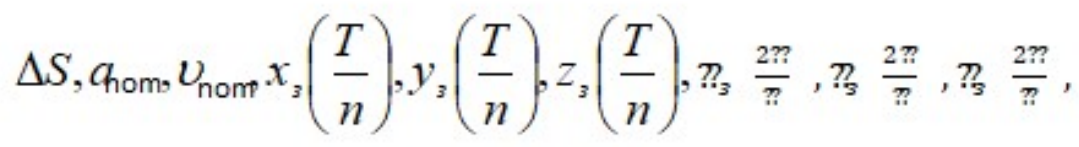

2) assignment: $x_{\mathrm{H}}\left(\frac{i T}{n}\right):=x_{3}\left(\frac{i T}{n}\right)-\Delta S ; x_{\mathrm{B}}\left(\frac{i T}{n}\right):=x_{3}\left(\frac{i T}{n}\right)+\Delta S$;

$$
\begin{aligned}
& y_{\mathrm{H}}\left(\frac{i T}{n}\right):=y_{3}\left(\frac{i T}{n}\right)-\Delta S ; y_{\mathrm{B}}\left(\frac{i T}{n}\right):=y_{3}\left(\frac{i T}{n}\right)+\Delta S ; \\
& Z_{\mathrm{H}}\left(\frac{i T}{n}\right):=z_{3}\left(\frac{i T}{n}\right)-\Delta S ; z_{\mathrm{B}}\left(\frac{i T}{n}\right):=z_{3}\left(\frac{i T}{n}\right)+\Delta S \\
& i=1, n ; l_{110}:=\frac{l_{1}}{n} ; l_{120}:=\frac{l_{2}}{n} ; l_{130}:=\frac{l_{3}}{n} ; l_{0}:=\max \left(l_{110}, l_{120}, l_{130}\right) ; \\
& t_{\mathrm{p} 1}:=\frac{v_{n o m}}{a_{n o m}} ; t_{\mathrm{T} 1}:=t_{\mathrm{p} 1} ; T_{1}:=\frac{l_{0}}{v_{n o m}}+\frac{1}{2} t_{\mathrm{p} 1}+\frac{1}{2} t_{\mathrm{T} 1} ; k_{\mathrm{p} 1}:=\frac{t_{\mathrm{p} 1}}{T_{1}} ; \\
& k_{\mathrm{T} 1}:=\frac{t_{\mathrm{T} 1}}{T_{1}} ; i:=1
\end{aligned}
$$

3) assignment: $t_{0}:=t$

4) Value update $x\left(t-t_{0}\right), y\left(t-t_{0}\right), z\left(t-t_{0}\right)$

5) verification of condition fulfillment: $x\left(\frac{i T}{n}\right)-x_{\mathrm{H}}\left(\frac{i T}{n}\right)<0$

if the condition is met, go to step 6 , then bypassing 7 to 8 ;

if the condition is not met, then go to step 7;

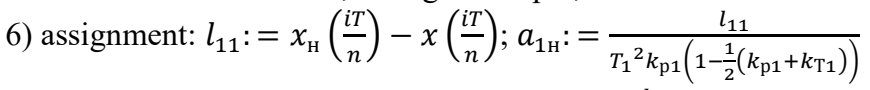

$$
a_{1 k}:=-a_{1 \mathrm{H}} ; v_{11}:=v_{1}+\frac{l_{11}}{T_{1}\left(1-\frac{1}{2}\left(k_{\mathrm{p} 1}+k_{\mathrm{T} 1}\right)\right)} ; v_{01}:=v_{1}
$$

7) assignment: $a_{1 \mathrm{H}}:=0 ; a_{1 k}:=0 ; v_{11}:=v_{1} ; v_{01}:=v_{1}$

8) verification of condition fulfillment: $y\left(\frac{i T}{n}\right)-y_{\mathrm{H}}\left(\frac{i T}{n}\right)<0$

if the condition is met, go to step 9, then bypassing 10 to 11 ;

if the condition is not met, then go to step 10;

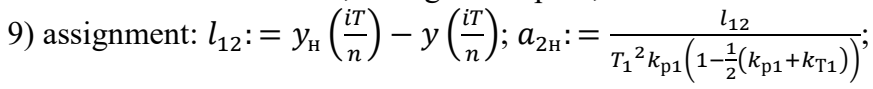




$$
a_{2 k}:=-a_{2 \mathrm{H}} ; v_{12}:=v_{2}+\frac{l_{12}}{T_{1}\left(1-\frac{1}{2}\left(k_{\mathrm{p} 1}+k_{\mathrm{T} 1}\right)\right)} ; v_{02}:=v_{2}
$$

10) assignment: $a_{2 \mathrm{H}}:=0 ; a_{2 k}:=0 ; v_{12}:=v_{2} ; v_{02}:=v_{2}$

11) verification of condition fulfillment: $z\left(\frac{i T}{n}\right)-Z_{\mathrm{H}}\left(\frac{i T}{n}\right)<0$

if the condition is met, go to step 12 , then bypassing 13 to 14 ;

if the condition is not met, then go to step 13;

12) assignment: $l_{13}:=z_{\mathrm{H}}\left(\frac{i T}{n}\right)-z\left(\frac{i T}{n}\right) ; a_{3 \mathrm{H}}:=\frac{l_{13}}{T_{1}{ }^{2} k_{\mathrm{p} 1}\left(1-\frac{1}{2}\left(k_{\mathrm{p} 1}+k_{\mathrm{T} 1}\right)\right.} ; a_{3 \mathrm{~K}}:=-a_{3 \mathrm{H}}$

$$
v_{13}:=v_{3}+\frac{l_{13}}{T_{1}\left(1-\frac{1}{2}\left(k_{\mathrm{p} 1}+k_{\mathrm{T} 1}\right)\right)} ; v_{03}:=v_{3}
$$

13) assignment: $a_{3 \mathrm{H}}:=0 ; a_{3 \mathrm{~K}}:=0 ; v_{13}:=v_{3} ; v_{03}:=v_{3}$

14) verification of condition fulfillment: $x\left(\frac{i T}{n}\right)-x_{\mathrm{B}}\left(\frac{i T}{n}\right)>0$

if the condition is met, go to step 15 , then bypassing 16 to 17 ;

if the condition is not met, then go to step 16;

15) assignment: $l_{11}:=x\left(\frac{i T}{n}\right)-x_{\mathrm{B}}\left(\frac{i T}{n}\right) ; a_{1 \mathrm{H}}:=-\frac{l_{11}}{T_{1}{ }^{2} k_{\mathrm{T} 1}\left(1-\frac{1}{2}\left(k_{\mathrm{p} 1}+k_{\mathrm{T} 1}\right)\right)}$

$$
a_{1 k}:=-a_{1 \mathrm{H}} ; v_{11}:=v_{1}-\frac{l_{11}}{T_{1}\left(1-\frac{1}{2}\left(k_{\mathrm{p} 1}+k_{\mathrm{T} 1}\right)\right)} ; v_{01}:=v_{1}
$$

16) assignment: $a_{1 \mathrm{H}}:=0 ; a_{1 k}:=0 ; v_{11}:=v_{1} ; v_{01}:=v_{1}$

17) verification of condition fulfillment: $y\left(\frac{i T}{n}\right)-y_{\mathrm{B}}\left(\frac{i T}{n}\right)>0$

if the condition is met, go to step 18 , then bypassing 19 to 20 ;

if the condition is not met, then go to step 19;

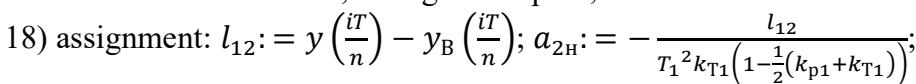

$$
a_{2 k}:=-a_{2 \mathrm{H}} ; v_{12}:=v_{2}-\frac{l_{12}}{T_{1}\left(1-\frac{1}{2}\left(k_{\mathrm{p} 1}+k_{\mathrm{T} 1}\right)\right)} ; v_{02}:=v_{2}
$$

19) assignment: $a_{2 \mathrm{H}}:=0 ; a_{2 k}:=0 ; v_{12}:=v_{2} ; v_{02}:=v_{2}$

20) verification of condition fulfillment: $z\left(\frac{i T}{n}\right)-z_{\mathrm{B}}\left(\frac{i T}{n}\right)>0$

if the condition is met, go to step 21, then bypassing 22 to 23 ;

if the condition is not met, then go to step 22;

21) assignment: $l_{13}:=z\left(\frac{i T}{n}\right)-z_{\mathrm{B}}\left(\frac{i T}{n}\right) ; a_{3 \mathrm{H}}:=-\frac{l_{13}}{T_{1}{ }^{2} k_{\mathrm{T} 1}\left(1-\frac{1}{2}\left(k_{\mathrm{p} 1}+k_{\mathrm{T} 1}\right)\right)} ; a_{3 k}:=-a_{3 \mathrm{H}}$

$$
v_{13}:=v_{3}-\frac{l_{13}}{T_{1}\left(1-\frac{1}{2}\left(k_{\mathrm{p} 1}+k_{\mathrm{T} 1}\right)\right)} ; v_{03}:=v_{3}
$$

22) assignment: $a_{3 \mathrm{H}}:=0 ; a_{3 k}:=0 ; v_{13}:=v_{3} ; v_{03}:=v_{3}$

$23)$ verification of condition fulfillment: $t-t_{0} \leq t_{\mathrm{p} 1}$

if the condition is met, go to steps 24,25 , then to 23 ;

if the condition is not met, then go to step 26;

24) assignment: $a_{1}:=a_{1 \mathrm{H}} ; a_{2}:=a_{2 \mathrm{H}} ; a_{3}:=a_{3 \mathrm{H}}$

25) Report acceleration $a_{1}, a_{2}, a_{3}$ using appropriate controls

26) verification of condition fulfillment: $t-t_{0} \leq T_{1}-t_{\mathrm{p} 1}$

if the condition is met, go to step 27,28 , then to 26 ;

if the condition is not met, then go to step 29;

27) $a_{1}:=0 ; a_{2}:=0 ; a_{3}:=0 ; v_{1}:=v_{11} ; v_{2}:=v_{12} ; v_{3}:=v_{13}$

28) Report acceleration $a_{1}, a_{2}, a_{3}$ and speed $v_{1}, v_{2}, v_{3}$ using appropriate controls

29) verification of condition fulfillment: $t-t_{0} \leq T_{1}$

if the condition is met, go to step 30,31 , then to 29 ;

if the condition is not met, then go to step 32;

30) assignment: $a_{1}:=a_{1 k} ; a_{2}:=a_{2 k} ; a_{3}:=a_{3 \kappa}$

31) Report acceleration $a_{1}, a_{2}, a_{3}$ using appropriate controls

32) assignment: $a_{1}:=0 ; a_{2}:=0 ; a_{3}:=0 ; v_{1}:=v_{01} ; v_{2}:=v_{02} ; v_{3}:=v_{03}$

33) Report acceleration $a_{1}, a_{2}, a_{3}$ and speed $v_{1}, v_{2}, v_{3}$ using appropriate controls

34) verification of condition fulfillment: $i<n$

if the condition is met, then go to step 3;

if the condition is not met, then no parameters are changed

Where $\Delta S$ - permissible deviation in each coordinate of the true trajectory from the given; $\boldsymbol{a}_{\text {nom }}, \boldsymbol{v}_{\text {nom }}-$ rated acceleration and speed of processor controls (controller) [7]; $\boldsymbol{x}_{3}\left(\frac{i T}{n}\right), \boldsymbol{y}_{3}\left(\frac{\boldsymbol{i} T}{n}\right), z_{3}\left(\frac{i T}{n}\right)-$ coordinate values of a given 
trajectory at certain time points $(i=\overline{\mathbf{1}, \boldsymbol{n}}) ; \quad \boldsymbol{x}_{\mathrm{H}}\left(\frac{i \boldsymbol{T}}{n}\right), \boldsymbol{x}_{\mathrm{B}}\left(\frac{i \boldsymbol{T}}{n}\right), \boldsymbol{y}_{\mathrm{H}}\left(\frac{i \boldsymbol{T}}{n}\right), \boldsymbol{y}_{\mathrm{B}}\left(\frac{i \boldsymbol{T}}{n}\right), \boldsymbol{z}_{\mathrm{H}}\left(\frac{i \boldsymbol{T}}{n}\right), \boldsymbol{z}_{\mathrm{B}}\left(\frac{i \boldsymbol{T}}{n}\right)-$ lower and upper boundaries of the permissible coordinates of the true path of the workpiece; $\boldsymbol{T}$ - time interval from the beginning of the movement to the end of the movement of the manipulation object; $\boldsymbol{n}$ - number of control points; $\boldsymbol{l}_{\mathbf{1 1 0}}, \boldsymbol{l}_{\mathbf{1 2 0}}, \boldsymbol{l}_{\mathbf{1 3 0}}$ - projections of the path between the control points on the corresponding axis; $\boldsymbol{l}_{\mathbf{0}}$ - maximum value of projection data; $\boldsymbol{t}_{\mathrm{p} 1}, \boldsymbol{t}_{\mathrm{T} 1}$ - acceleration and braking time on the path section, if it is necessary to change the workpiece position (correction section) due to the large difference between the truth and the given path [8]; $\boldsymbol{k}_{\mathrm{p} \mathbf{1}}, \boldsymbol{k}_{\mathrm{T} \mathbf{1}}-$ acceleration and braking factors in the specified area; $i$ - control point number; $\boldsymbol{t}_{\mathbf{0}}$ - start time of movement in the adjustment section; $\boldsymbol{x}\left(\boldsymbol{t}-\boldsymbol{t}_{\mathbf{0}}\right), \boldsymbol{y}\left(\boldsymbol{t}-\boldsymbol{t}_{\mathbf{0}}\right), \boldsymbol{z}\left(\boldsymbol{t}-\boldsymbol{t}_{\mathbf{0}}\right)$ - true coordinates of the workpiece trajectory at certain time points; $a_{j \mathrm{H}}, \boldsymbol{a}_{j k}-$ initial and final acceleration along the corresponding coordinate axis in the correction section $(\boldsymbol{j}=\overline{\mathbf{1}, \mathbf{3}}) ; v_{1 j}$ - speed along the corresponding coordinate axis in the correction section; $v_{0 j}$ - speed along the corresponding coordinate axis before starting corrections [9]; $l_{1 j}$ - length of the correction section. The speed in the correction section along the $j$ axis increases or decreases by $\frac{\boldsymbol{l}_{1 j}}{\boldsymbol{T}_{\mathbf{1}}\left(\mathbf{1}-\frac{\mathbf{1}}{2}\left(\boldsymbol{k}_{\mathrm{p} 1}+\boldsymbol{k}_{\mathrm{T} 1}\right)\right)}$, and acceleration is equal to $\pm \frac{\boldsymbol{l}_{1 j}}{\boldsymbol{T}_{\mathbf{1}}{ }^{2} \boldsymbol{k}_{\mathrm{p} 1}\left(\mathbf{1}-\frac{1}{2}\left(\boldsymbol{k}_{\mathrm{p} 1}+\boldsymbol{k}_{\mathrm{T} 1}\right)\right)}$ [10]. Time $\boldsymbol{T}_{\mathbf{1}}$, necessary to adjust the workpiece position is determined from the formula $l_{i}=v_{i}\left(T-\frac{1}{2} t_{\mathrm{P}}-\frac{1}{2} t_{\mathrm{T}}\right)$ [10]. At the same time, length $l_{i}$ is taken as maximum of the possible movements in the correction area.

\section{Summary}

To create the manipulator drive control program that provides capture of the manipulation object on the basis of the functional model for controlling capture of the manipulation object previously developed [1], we will draw up an algorithm for the system functioning in fulfilling the specified purpose.

The resulting algorithm has the following form:

1) input: $\phi_{1 \mathrm{H}}, \phi_{2 \mathrm{H}}, \phi_{3 \mathrm{H}}, \phi_{1 \mathrm{~B}}, \phi_{2 \mathrm{~B}}, \phi_{3 \mathrm{~B}}, x_{\mathrm{HO}}, y_{\mathrm{HO}_{0}}, z_{\mathrm{HO}}, x_{\mathrm{BO}}, y_{\mathrm{BO}}, z_{\mathrm{BO}}, v_{n o m}, \omega_{n o m}, \Delta t$

2) verification of condition fulfillment: $x_{\mathrm{Hо}}-x_{\mathrm{H}}<0$

if the condition is met, then go to steps 3,4 , then bypassing $5,6,7$ to 8 ;

if the condition is not met, then go to steps $5,6,7$, then return to 2

3) assignment: $v_{1}:=0$

4) report speed $v_{1}$ to the appropriate controls

5) assignment: $v_{1}:=v_{\text {nom }}$

6) report speed $v_{1}$ to the appropriate controls

7) value update $x_{\mathrm{H}}, y_{\mathrm{H}}, z_{\mathrm{H}}, x_{\mathrm{B}}, y_{\mathrm{B}}, z_{\mathrm{B}}$ according to the additional locator and value update $\phi_{1}, \phi_{2}, \phi_{3}$ according to technical vision

8) verification of condition fulfillment: $y_{\mathrm{но}}-y_{\mathrm{H}}<0$

if the condition is met, then go to steps 9,10 , then bypassing $11,12,13$ to 14 ;

if the condition is not met, then go to steps $11,12,13$, then return to 8

9) assignment: $v_{2}:=0$

10) report speed $v_{2}$ to the appropriate controls

11) assignment: $v_{2}:=v_{\text {nom }}$

12) report speed $v_{2}$ to the appropriate controls

13) value update $x_{\mathrm{H}}, y_{\mathrm{H}}, z_{\mathrm{H}}, x_{\mathrm{B}}, y_{\mathrm{B}}, z_{\mathrm{B}}$ according to the additional locator and value update $\phi_{1}, \phi_{2}, \phi_{3}$ according to technical vision

14) verification of condition fulfillment: $z_{\mathrm{Hо}}-z_{\mathrm{H}}<0$

if the condition is met, then go to steps 15,16 , then bypassing $17,18,19$ to 20 ;

if the condition is not met, then go to steps $17,18,19$, then return to 14

15) assignment: $v_{3}:=0$

16) report speed $v_{3}$ to the appropriate controls

17) assignment: $v_{3}:=v_{\text {nom }}$

18) report speed $v_{3}$ to the appropriate controls

19) value update $x_{\mathrm{H}}, y_{\mathrm{H}}, z_{\mathrm{H}}, x_{\mathrm{B}}, y_{\mathrm{B}}, z_{\mathrm{B}}$ according to the additional locator and value update $\phi_{1}, \phi_{2}, \phi_{3}$ according to technical vision

20) verification of condition fulfillment: $x_{\text {во }}-x_{\mathrm{B}}>0$

if the condition is met, then go to steps 21,22 , then bypassing $23,24,25$ to 26 ;

if the condition is not met, then go to steps $23,24,25$, then return to 20

21) assignment: $v_{1}:=0$

22) report speed $v_{1}$ to the appropriate controls

23) assignment: $v_{1}:=-v_{\text {nom }}$

24) report speed $v_{1}$ to the appropriate controls

25) value update $x_{\mathrm{H}}, y_{\mathrm{H}}, z_{\mathrm{H}}, x_{\mathrm{B}}, y_{\mathrm{B}}, z_{\mathrm{B}}$ according to the additional locator and value update $\phi_{1}, \phi_{2}, \phi_{3}$ according to technical vision

26) verification of condition fulfillment: $y_{\text {во }}-y_{\mathrm{B}}>0$ 
if the condition is met, then go to steps 27,28 , then bypassing $29,30,31$ to 32 ;

if the condition is not met, then go to steps $29,30,31$, then return to 26

27) assignment: $v_{2}:=0$

28) report speed $v_{2}$ to the appropriate controls

29) assignment: $v_{2}:=-v_{\text {nom }}$

30) report speed $v_{2}$ to the appropriate controls

31) value update $x_{\mathrm{H}}, y_{\mathrm{H}}, z_{\mathrm{H}}, x_{\mathrm{B}}, y_{\mathrm{B}}, z_{\mathrm{B}}$ according to the additional locator and value update $\phi_{1}, \phi_{2}, \phi_{3}$ according to technical vision

32) verification of condition fulfillment: $z_{b o}-z_{b}>0$

if the condition is met, then go to steps 33,34 , then bypassing $35,36,37$ to 38 ;

if the condition is not met, then go to steps $35,36,37$, then return to 32

33) assignment: $v_{3}:=0$

34) report speed $v_{3}$ to the appropriate controls

35) assignment: $v_{3}:=-v_{\text {nom }}$

36) report speed $v_{3}$ to the appropriate controls

37) value update $x_{\mathrm{H}}, y_{\mathrm{H}}, z_{\mathrm{H}}, x_{\mathrm{B}}, y_{\mathrm{B}}, z_{\mathrm{B}}$ according to the additional locator and value update $\phi_{1}, \phi_{2}, \phi_{3}$ according to technical vision

38) verification of condition fulfillment: $\phi_{1 \mathrm{H}}-\phi_{1}<0$

if the condition is met, then go to steps 33,34 , then bypassing $35,36,37$ to 38 ;

if the condition is not met, then go to steps $5,6,7$, then return to 2

39) assignment: $\omega_{1}:=0$

40) report speed $v_{1}$ to the appropriate controls

41) assignment: $\omega_{1}:=\omega_{\text {nom }}$

42) report speed $\omega_{1}$ to the appropriate controls

43) value update $x_{\mathrm{H}}, y_{\mathrm{H}}, z_{\mathrm{H}}, x_{\mathrm{B}}, y_{\mathrm{B}}, z_{\mathrm{B}}$ according to the additional locator and value update $\phi_{1}, \phi_{2}, \phi_{3}$ according to technical vision

44) verification of condition fulfillment: $\phi_{2 \mathrm{H}}-\phi_{2}<0$

if the condition is met, then go to steps 45,46 , then bypassing 47, 48, 49 to 50;

if the condition is not met, then go to steps $47,48,49$, then return to 44

45) assignment: $\omega_{2}:=0$

46) report speed $\omega_{2}$ to the appropriate controls

47) assignment: $\omega_{2}:=\omega_{\text {nom }}$

48) report speed $\omega_{2}$ to the appropriate controls

49) value update $x_{\mathrm{H}}, y_{\mathrm{H}}, z_{\mathrm{H}}, x_{\mathrm{B}}, y_{\mathrm{B}}, z_{\mathrm{B}}$ according to the additional locator and value update $\phi_{1}, \phi_{2}, \phi_{3}$ according to technical vision

50) verification of condition fulfillment: $\phi_{3 \mathrm{H}}-\phi_{3}<0$

if the condition is met, then go to steps 51,52 , then bypassing $53,54,55$ to 56 ;

if the condition is not met, then go to steps $53,54,55$, then return to 50

51) assignment: $\omega_{3}:=0$

52) report speed $\omega_{3}$ to the appropriate controls

53) assignment: $\omega_{3}:=\omega_{\text {nom }}$

54) report speed $\omega_{3}$ to the appropriate controls

55) value update $x_{\mathrm{H}}, y_{\mathrm{H}}, z_{\mathrm{H}}, x_{\mathrm{B}}, y_{\mathrm{B}}, z_{\mathrm{B}}$ according to the additional locator and value update $\phi_{1}, \phi_{2}, \phi_{3}$ according to technical vision

56) verification of condition fulfillment: $\phi_{1 b}-\phi_{1}>0$

if the condition is met, then go to steps 57,58 , then bypassing $59,60,61$ to 62 ;

if the condition is not met, then go to steps $59,60,61$, then return to 56

57) assignment: $\omega_{1}:=0$

58) report speed $\omega_{1}$ to the appropriate controls

59) assignment: $\omega_{1}:=-\omega_{\text {nom }}$

60) report speed $\omega_{1}$ to the appropriate controls

61) value update $x_{\mathrm{H}}, y_{\mathrm{H}}, z_{\mathrm{H}}, x_{\mathrm{B}}, y_{\mathrm{B}}, z_{\mathrm{B}}$ according to the additional locator and value update $\phi_{1}, \phi_{2}, \phi_{3}$ according to technical vision

62) verification of condition fulfillment: $\phi_{2 b}-\phi_{2}>0$

if the condition is met, then go to steps 63,64 , then bypassing $65,66,67$ to 68 ;

if the condition is not met, then go to steps $65,66,67$, then return to 62

63) assignment: $\omega_{2}:=0$

64) report speed $\omega_{2}$ to the appropriate controls

65) assignment: $\omega_{2}:=-\omega_{\text {nom }}$

66) report speed $\omega_{2}$ to the appropriate controls 
67) value update $x_{\mathrm{H}}, y_{\mathrm{H}}, z_{\mathrm{H}}, x_{\mathrm{B}}, y_{\mathrm{B}}, z_{\mathrm{B}}$ according to the additional locator and value update $\phi_{1}, \phi_{2}, \phi_{3}$ according to technical vision

68) verification of condition fulfillment: $\phi_{3 b}-\phi_{3}>0$

if the condition is met, then go to steps 69,70 , then bypassing $71,72,73$ to 74 ;

if the condition is not met, then go to steps $71,72,73$, then return to 68

69) assignment: $\omega_{3}:=0$

70) report speed $\omega_{3}$ to the appropriate controls

71) assignment: $\omega_{3}:=-\omega_{\text {nom }}$

72) report speed $\omega_{3}$ to the appropriate controls

73) value update $x_{\mathrm{H}}, y_{\mathrm{H}}, z_{\mathrm{H}}, x_{\mathrm{B}}, y_{\mathrm{B}}, z_{\mathrm{B}}$ according to the additional locator and value update $\phi_{1}, \phi_{2}, \phi_{3}$ according to technical vision

74) assignment: $v_{4}:=v_{\text {nom }} ; t_{0}:=t$

75) report speed $v_{4}$ to the appropriate controls

76) verification of condition fulfillment: $t-t_{0} \geq \Delta t$

if the condition is met, then go to step 78,78

if the condition is not met, then go to step 76

77) assignment: $v_{4}:=0$

78) Report speed $v_{4}$ to the appropriate controls

Where $\phi_{1 \mathrm{H}}, \phi_{2_{\mathrm{H}}}, \phi_{3_{\mathrm{H}}}, \phi_{1_{\mathrm{B}}}, \phi_{2_{\mathrm{B}}}, \phi_{3_{\mathrm{B}}}$ - lower and upper boundaries of acceptable values of rotation angles of the workpiece relative to the coordinate axes before its capture; $\boldsymbol{x}_{\mathrm{Hо}}, \boldsymbol{y}_{\mathrm{Hо}}, \boldsymbol{z}_{\mathrm{Ho}}, \boldsymbol{x}_{\mathrm{во}}, \boldsymbol{y}_{\mathrm{во}}, \boldsymbol{z}_{\mathrm{во}}$ - lower and upper boundaries of the area on each coordinate axis, in which the workpiece shall be located before it is captured; $\boldsymbol{x}_{\mathrm{H}}, \boldsymbol{y}_{\mathrm{H}}, \boldsymbol{z}_{\mathrm{H}}, \boldsymbol{x}_{\mathrm{B}}, \boldsymbol{y}_{\mathrm{B}}, \boldsymbol{z}_{\mathrm{B}}$ - lower and upper boundaries of the area along each coordinate axis, in which the workpiece is located at a given time; $\boldsymbol{\phi}_{1}, \boldsymbol{\phi}_{2}, \boldsymbol{\phi}_{3}$ - true values of rotation angles of the workpiece relative to the coordinate axes; $\boldsymbol{v}_{\text {ном }}-$ rated speed of progressive controls; $\boldsymbol{\omega}_{\text {nom }}$ - rated speed of rotational controls; $\boldsymbol{v}_{1}, \boldsymbol{v}_{2}, \boldsymbol{v}_{3}$ - speed of controls for portable degrees of mobility; $\boldsymbol{\omega}_{\mathbf{1}}, \boldsymbol{\omega}_{2}, \boldsymbol{\omega}_{3}$-speed of controls, orienting rotational degrees of mobility; $\boldsymbol{v}_{\mathbf{4}}-$ speed of controls, orienting translational degree of mobility; $\Delta t$ - time required for the workpiece capture.

In this algorithm, giving speed $\boldsymbol{v}_{4}:=\boldsymbol{v}_{\text {nom }}$ the moving jaw of the gripper, and then zeroing this speed after a certain time period $\Delta t$ is the process of workpiece capturing.

Based on this algorithm, a drive control program can be compiled to capture the manipulated object.

\section{Conclusions}

The following algorithms have been developed: functioning of the manipulator drive control system using the processor in order to ensure the workpiece movement along a predetermined path, in order to eliminate the deviation of the true path of the manipulated object from the predetermined one and ensure the capture of the manipulated object.

The following algorithms were used at their creation: control method by speed vector, method of sequential adjustments. The last algorithm is based on a change in the grasp position when the generalized coordinates of the workpiece (axial coordinates and rotation angles) go beyond the allowable area.

These algorithms can be used to create the manipulator control programs.

\section{Acknowledgements}

The work is performed according to the Russian Government Program of Competitive Growth of Kazan Federal University.

\section{References}

[1] Fardeev, A.R. Functional models of the operation of manipulator systems with three translational portable degrees of mobility / A.R. Fardeev, R.G. Mardanshin, A.A. Fardeev, A.M. Abdullina // Scientific and Technical Bulletin of the Volga Region - 2018. - No. 12. - P. 122-125

[2] Krainev, A.F. Dictionary-reference on mechanisms / Krainev A.F. - M. : Mashynostroeniye, 1987. — 560 p.

[3] Fardeev, A.R. The functioning of the control system for the manipulation object movement along a given path / A.R. Fardeev, A.A. Fardeev, G.M. Safina // Modern technologies in mechanical engineering: Collection of Articles of the XIX International Scientific-Practical Conference, Penza, December 2015 / ANOO "Volga House of Knowledge" - Penza. - 2015. - P. 107 - 111

[4] T.S. Jagadevlah, R.T. Smith, "Generation schemes for wind power plants", paper presented in the 10th Intersociety Energy Conversion Engineering Conference (IECEC), Newark.: Delaware, August, 1975.

[5] F.D. Bairamov, I.G. Mardamshin, "Investigation of operation of hydraulic systems with wind pumping units", Russian Aeronautics, vol. 51, № 3, pp. 314-320, 2008.

[6] Nikitin, N. N. The course of theoretical mechanics / Nikitin N. N. - M.: Vysshaya Shkola, 1990. - 608 p.

[7] Farit D. Bairamov, Bulat F. Bairamov, "About hybrid system stability with distributed and concentrated parameters”, Helix, vol. 8, № 1, pp. 2493-2498, 2018. 
[8] K. Peiffer, N. Rouche, "Liapunov’s second method applied to partial stability”, J. de Mech., vol. 8, № 2, pp. 323-334, 1969.

[9] T. Yoshizawa, “Stability theory by Lyapunov's second method”, Tokyo: Math. Soc. Jap., 223 p., 1966.

[10] Fardeev, A.R. The functioning of the control system of the manipulation object movement at the deviation of the actual trajectory from a given one / A. Fardeev, A.A. Fardeev // Modern information technologies in quality management: Collection of Articles of the VI International Scientific and Applied Conference, Penza, June 2018 / ANOO "Volga House of Knowledge" - Penza. - 2018. - P. 95 - 98 